\title{
Aminoglycoside-induced hearing loss in HIV-positive and HIV- negative multidrug-resistant tuberculosis patients
}

\author{
Tashneem Harris, Soraya Bardien, H Simon Schaaf, Lucretia Petersen, Greetje de Jong, Johannes J Fagan
}

Background. Ototoxicity following aminoglycoside treatment for multidrug-resistant tuberculosis (MDR-TB), is a significant problem. This study documents the incidence of ototoxicity in HIVpositive and HIV-negative patients with MDR-TB and presents clinical guidelines relating to ototoxicity.

Methods. A prospective cohort study of 153 MDR-TB patients with normal hearing and middle ear status at baseline controlling for 6 mitochondrial mutations associated with aminoglycosiderelated ototoxicity, at Brooklyn Chest Hospital in Cape Town. Pure tone audiometry was performed monthly for 3 months to determine hearing loss. HIV status was recorded, as was the presence of 6 mutations in the MT-RNR1 gene.
Results. Fifty-seven per cent developed high-frequency hearing loss. HIV-positive patients (70\%) were more likely to develop hearing loss than HIV-negative patients (42\%). Of 115 patients who were genetically screened, none had MT-RNR1 mutations.

Conclusion. Ototoxic hearing loss is common in MDR-TB patients treated with aminoglycosides. HIV-positive patients are at increased risk of ototoxicity. Auditory monitoring and auditory rehabilitation should be an integral part of the package of care of MDR-TB patients.

S Afr Med J 2012;102(6):363-366.
Multidrug-resistant tuberculosis (MDR-TB, i.e. Mycobacterium tuberculosis resistant to both isoniazid and rifampicin with or without other drug resistance) is an increasing problem, especially in sub-Saharan Africa, ${ }^{1}$ where the significant increase in MDR-TB has been linked to the human immunodeficiency virus (HIV) epidemic. ${ }^{1}$

World Health Organization (WHO) guidelines recommend second-line injectable drugs (amikacin, kanamycin, capreomycin) for MDR-TB treatment. ${ }^{2}$ Patients are on long-term treatment plans including a second-line injectable drug for at least 6 months, or 4 months after sputum culture conversion (i.e. 2 sputum cultures negative 1 month apart). These drugs have significant adverse effects, especially ototoxicity and nephrotoxicity. Unlike nephrotoxicity, ototoxicity is permanent. ${ }^{3}$ Risk factors include cumulative drug dose, duration of treatment, bacteraemia, renal or liver failure, and concomitant administration of drugs such as loop diuretics, that have a synergistic ototoxic effect. ${ }^{4}$

Some individuals may develop sudden profound sensorineural hearing loss following a single dose of aminoglycoside. This may be associated with a genetic susceptibility caused by the 1555A

Department of Otolaryngology, Kimberley Hospital Complex; and Division of Otolaryngology, University of Cape Town Medical School Tashneem Harris, MB ChB, FCORL (SA)

Division of Molecular Biology and Human Genetics, Stellenbosch University Soraya Bardien, BSc, PhD (Human Genetics)

Greetje de Jong, MB ChB, BSc (Hons) (Hum Genet), MMed, MD

Department of Paediatrics and Child Health, Stellenbosch University H Simon Schaaf, MB ChB, DCM, MMed (Paed), MD (Paed)

Division of Communication Sciences and Disorders, University of Cape Town Medical School

Lucretia Petersen, B Speech Therapy and Audiology, MSc Audiology

Division of Otolaryngology, University of Cape Town Medical School Johannes J Fagan, MB ChB, FCS (SA), MMed (Otol) to $G$ substitution on the mitochondrial $12 S$ rRNA. ${ }^{5}$ Genetic predisposition may be predicted by identification of mutations in the MT-RNR1 gene.,6 The literature suggests that mitochondrial mutations in the $12 \mathrm{~S}$ rRNA are rare $(0.09 \%-3.96 \%)$, depending on the population studied. ${ }^{7,8}$ Owing to the rarity of these mutations, genetic predisposition may be unlikely to contribute significantly to the excessive risk of ototoxicity seen in developing countries. Other environmental factors may cause patients to be at increased risk. This study was divided into 2 parts of which the first relates to prevalence of the 6 known aminoglycoside-induced deafness mutations in the MT-RNR1 gene in a cohort of MDR-TB patients. ${ }^{5,6}$ Although these data have been published, they are cited where referred to in the text for completeness. The second part involves prospective hearing evaluation.

The high incidence of MDR-TB in South Africa and lack of efficacious alternative drugs mean that increasing numbers of people are at risk of developing aminoglycoside- or polypeptide-related ototoxicity. The lack of audiological monitoring results in ototoxicity going undetected. No data on the incidence of aminoglycosideinduced deafness have been documented for South Africa.

The study aimed to: (i) document the incidence and audiological profile of aminoglycoside-induced hearing loss in HIV-positive and HIV-negative patients with MDR-TB; and (ii) formulate guidelines on the use of aminoglycosides in MDR-TB treatment relating to ototoxicity.

\section{Methods}

MDR-TB patients were recruited at Brooklyn Chest Hospital (BCH) in Cape Town, a dedicated 349-bed public sector TB hospital, that has received several wards for the management of MDR-TB patients.

On admission, all patients whose HIV status is unknown are routinely tested for HIV after informed consent and pre- and post-test counselling. Antiretroviral treatment is initiated when appropriate as part of comprehensive care.

Only patients with normal hearing on hospital admission and receiving aminoglycosides as part of their treatment within 3 months of hospitalisation were included. Serum aminoglycoside levels were not recorded. Patients underwent pure tone audiometry (PTA) and tympanometry on admission, then monthly for 3 months. Pure tone audiometry, including air and bone conduction threshold 
measurements between $250 \mathrm{~Hz}$ and $8000 \mathrm{~Hz}$, was performed in a sound-treated room. Ototoxic threshold shift from the initial baseline audiogram was defined as: $(1) \geq 20$ decibel $(\mathrm{dB})$ decrease at any one test frequency, (2) $\geq 10 \mathrm{~dB}$ decrease at any 2 adjacent frequencies, or (3) loss of response at 3 consecutive frequencies where responses had previously been obtained. The severity of hearing loss was graded as: (1) mild: PTA 26 - $40 \mathrm{~dB}$ hearing level (HL), (2) moderate: PTA 41 - 55 dB HL, (3) moderately severe: PTA 56 - $70 \mathrm{~dB}$ HL, (4) severe: PTA 71 - $90 \mathrm{~dB}$ HL, and (5) profound: PTA $>90 \mathrm{~dB}$ HL. Age, gender, history of noise exposure, specific aminoglycoside therapy, presence of tinnitus, HIV status and whether patients were on combination antiretroviral therapy (cART) were recorded. Although pre-treatment baseline audiometry could not be performed on patients commenced on aminoglycosides by TB clinics prior to admission, only patients with normal hearing on admission were included. As part of the genetics arm of the study, peripheral blood samples were collected in EDTA-coated tubes from each study participant, and genetic testing was performed to screen for the 6 known aminoglycoside-induced deafness mutations in the MT-RNR1 gene (A1555G, C1494T, A827G, T1291C, T1095C and 961 indelC) using the new SNAPshot technique developed by the Tygerberg Genetics Department. Polymerase chain reaction (PCR) primers and SnapShot primers were specially designed that spanned the entire length of the MT-RNR1 gene. The SnapShot technique can detect the 6 known mutations in a single reaction. ${ }^{5,6}$

The $t$-test two-sample assuming unequal variances test was used to calculate the difference in the mean ages between HIV-positive and HIV-negative patients with hearing loss at a 95\% confidence interval (CI). The odds ratio (OR) $(95 \% \mathrm{CI})$ was used to calculate the measure of association of HIV and aminoglycoside-induced hearing loss. Fisher's exact test was used to determine whether there were associations between gender and hearing loss, and to calculate whether there was a significant difference between HIV status and the severity of hearing loss.

The study was approved by the Committee for Human Research at Stellenbosch University (protocol number: N05/09/165) and the Research Ethics Committee at the University of Cape Town (ref: 443/2005).

\section{Results}

We studied 153 patients; 2 refused HIV testing and were excluded. The median age was 36 years (range 14 - 70 years). All patients were on long-term treatment plans with second-line injectable agents; 145 (96\%) had received kanamycin, 5 (3\%) streptomycin and 1 (1\%) capreomycin.

Of 151 patients, 86 (57\%) were HIV-positive. All HIV-positive patients were on cART, which comprised a combination of 2 nucleoside reverse transcriptase inhibitors (NRTIs) and a nonnucleoside reverse transcriptase inhibitor (NNRTI) or a protease inhibitor (PI).

Table 1 summarises hearing loss following 3 months of in-hospital aminoglycoside treatment; 87 (58\%) subjects developed hearing loss

Table 1. Frequency of hearing loss after 3 months on aminoglycoside treatment for MDR-TB, and relation to gender

\begin{tabular}{llll}
\hline Gender & $\begin{array}{l}\text { MDR-TB cases } \\
\boldsymbol{n}(\%)\end{array}$ & $\begin{array}{l}\text { Hearing loss } \\
\boldsymbol{n}(\%)\end{array}$ & $\begin{array}{l}\boldsymbol{p} \text {-value (female } \\
\text { v. male) }\end{array}$ \\
\hline All cases & 151 & $87(58)$ & \\
Female & $100(66)$ & $55(55)$ & $p=0.5$ \\
Male & $51(34)$ & $32(65)$ &
\end{tabular}

that involved the higher frequencies $(4000-8000 \mathrm{~Hz}$ ) in all patients. In $5(6 \%)$ patients with hearing loss, it progressed to involve lower frequencies (500, 1000,2000 and $3000 \mathrm{~Hz}) ; 13(15 \%)$ developed asymmetrical hearing loss, and 11 (13\%) complained of tinnitus.

Of patients who developed hearing loss, 74 (85\%) had acquired MDR-TB and had received streptomycin in the preceding 6 months; 13 (15\%) had new MDR-TB (i.e. no previous anti-TB treatment or treatment for $<1$ month). Gender did not correlate with developing hearing loss $(p=0.5$; Table 1$)$.

HIV-positive patients $(60 / 86 ; 70 \%)$ were more likely to develop hearing loss than HIV-negative patients $(27 / 65 ; 42 \%$, OR $3.25,95 \%$ CI $1.65-6.37 ; p<0.001)$. Fig. 1 shows the age distribution. There was no significant difference between the ages of the two groups. The median age (standard deviation) of HIV-positive patients with sensorineural hearing loss was 34 (9.1) years, while HIV-negative patients had a median age of 40 (13.7) years. There was no association between HIV status and severity of hearing loss (Table 2). Patients who developed ototoxicity were mainly in the 30 - 39-year-old age group, which was representative of the study population's age distribution.

None of 115 patients who consented to genetic testing had mitochondrial mutations. Of the 87 patients who developed hearing loss, 38 (44\%) refused genetic testing, which makes assessment of the role of mutations in hearing loss equivocal.

\section{Discussion}

Aminoglycoside ototoxicity targets the sensory neuroepithelium of the cochlea. ${ }^{3}$ Loss of cochlear hair cells results in secondary degeneration of the auditory nerve. ${ }^{3}$ Outer hair cells are more susceptible than inner hair cells. Labyrinthine injury is usually gradual, progressive, symmetrically bilateral and permanent. ${ }^{3}$ However, as in our study, asymmetrical losses do occur. ${ }^{3}$ Ototoxic injury and associated hearing loss may progress for weeks following cessation of aminoglycoside treatment, owing to its long half-life in cochlear tissue. ${ }^{3}$ The basal region of the cochlea is more susceptible to injury than the apical region. This 'cochleotopic' gradient of susceptibility is expressed as high-frequency hearing loss, which extends to include progressively lower frequencies with more extensive cochlear damage. ${ }^{9}$ This is consistent with our finding that hearing loss, as documented by PTA, involved mainly higher frequencies $(4000-8000 \mathrm{~Hz})$. In $6 \%$ of patients, this progressed to involve lower frequencies $(500,1000$, 2000 and $3000 \mathrm{~Hz}$ ), consequently affecting speech comprehension.

Within 3 months of in-hospital aminoglycoside therapy, 57\% of patients developed high-frequency hearing loss. Torun et al. reported ototoxicity in $42 \%$ of MDR-TB patients occurring at $4.7 \pm 1.7$ months

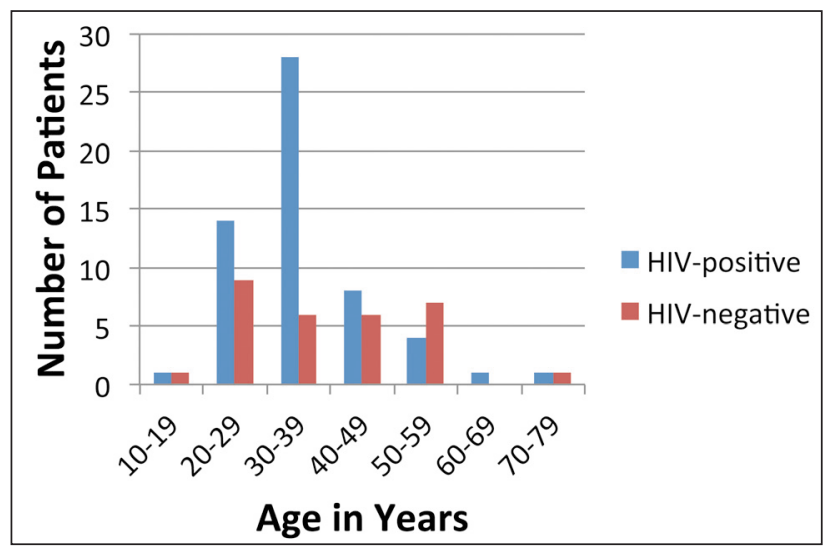

Fig. 1. Age distribution of HIV-positive and HIV-negative patients treated with aminoglycosides for multidrug-resistant $T B$ who developed ototoxic hearing loss. 
Table 2. Severity of hearing loss in HIV-positive and HIV-negative multidrug-resistant TB patients treated with aminoglycosides

\begin{tabular}{lccc}
\hline & \multicolumn{3}{c}{ Severity of hearing loss } \\
\cline { 2 - 3 } HIV status & Mild - moderate (PTA 26 - 55 dB HL) & Moderate - severe (PTA 56 - 71 dB HL) & Severe - profound (PTA >71 dB HL) \\
\hline HIV-positive $(n=86)$ & $20(23 \%)$ & 0 & $40(47 \%)$ \\
HIV-negative $(n=65)$ & $7(10 \%)$ & 0 & $20(30 \%)$
\end{tabular}

of treatment. ${ }^{10}$ This frequent and early occurrence of ototoxicity may be due to extended exposure to aminoglycosides, as $40 \%$ of patients had previously been exposed to streptomycin..$^{10}$ This result is similar to our study where $85 \%$ of patients had previously been exposed to streptomycin, and with findings that showed an association between ototoxicity and cumulative dose of aminoglycoside. ${ }^{4}$

No reported studies on aminoglycoside-induced ototoxicity in MDR-TB have included significant numbers of HIV-positive patients. Anti-TB treatment is frequently administered in HIVpositive patients, including MDR-TB treatment. MDR-TB co-infected patients tend to have poorer outcomes and significant mortality in the first few months of MDR-TB treatment. ${ }^{1}$ Some studies, conducted in HIV-negative MDR-TB patients who were hospitalised and had adverse effects monitored for the duration of treatment, showed no compromise in cure when aminoglycosides were stopped and the treatment regimen changed when patients developed ototoxicity. ${ }^{9,10}$ Consequently, in our setting, aminoglycosides are generally continued in HIV-positive patients co-infected with MDR-TB, despite the risk of developing hearing loss.

Evidence suggesting that cART may be ototoxic is mainly based on cross-sectional studies and case reports, and further studies are needed to establish these effects. ${ }^{11}$ In our study (the first to look at incidence of hearing loss in HIV-positive patients on cART and MDR-TB treatment), HIV-positive patients had a more than 3 times greater risk of developing ototoxic hearing loss.

Ototoxicity is diagnosed by comparing an initial audiogram ideally obtained before initiation of ototoxic drugs - with hearing thresholds using serial audiograms, particularly ultra-high frequency thresholds. ${ }^{12}$ Patients receiving ototoxic antibiotics should be monitored weekly or biweekly. ${ }^{12}$ Oto-acoustic emissions (OAEs) are more sensitive at detecting auditory dysfunction than high-frequency pure-tone audiometry and have been used to monitor ototoxicity. ${ }^{12}$ Owing to the long half-life of aminoglycosides in cochlear tissues, patients should be monitored for up to about 6 months following completion of MDR therapy. ${ }^{9}$ Although hearing loss was detected in $57 \%$ of our patients, this figure could be greater if a more sensitive monitoring method such as high-frequency audiometry or OAE had been used, or if patients were followed up until after completion of aminoglycoside therapy. Once patients are discharged from Brooklyn Chest Hospital, they often cannot report back to the hospital for audiometric evaluation; consequently, because of the paucity of audiological services at peripheral clinics, monitoring for hearing loss following discharge from the hospital is non-existent.

Aminoglycoside treatment has commenced in the community owing to insufficient beds at TB hospitals, and hence the need to decentralise audiological services to TB clinics. The serious lack of audiological testing facilities, hearing aids and audiological rehabilitation in the Western Cape, is compromising MDR-TB care for patients who have become deaf as a consequence of ototoxic drug therapy. ${ }^{13}$

A study of patients' awareness of ototoxicity with MDR-TB treatment reported that only $20 \%$ were aware that their treatment had ototoxic adverse effects, and that no patients on MDR-TB treatment were being monitored for ototoxicity. ${ }^{14}$ Even though more than half of the patients reported auditory symptoms since the commencement of treatment, none had been referred to an audiologist or otolaryngologist for management, despite almost half of them reporting that these symptoms affected their daily lives. ${ }^{14}$

When monitoring for ototoxicity, the aim is to detect hearing loss before it affects speech frequencies and the patient develops a communication problem. The treatment regimen can then be changed by either replacing the ototoxic drug or stopping it. MDR-TB is a life-threatening disease, and in most cases it may not be possible to safely alter the treatment without compromising cure of the patient. However, maintaining communication remains a major quality-of-life issue for patients. Therefore, the purpose of monitoring for ototoxicity is to assist the patient with communication strategies or amplification in the form of hearing aids when hearing loss occurs; it may also include tinnitus counselling. Managing MDR-TB patients with a holistic approach requires screening and monitoring for ototoxicity as part of the package of care of MDR-TB and possibly integration into national TB control programmes.

\section{Conclusion}

A high rate of hearing loss in patients on aminoglycoside-containing MDR-TB treatment was shown. The report is the first to show an association between HIV infection and ototoxicity in patients on MDR-TB treatment. Further studies are required to elucidate the mechanism of increased ototoxicity in HIV-positive patients. Hearing loss is likely to increase, given the high prevalence of HIV infection in South Africa, and information regarding possible hearing loss should be included as part of informed consent before commencing aminoglycoside therapy.

Acknowledgements. This study was funded by the South African Society of Otorhinolaryngology, Head and Neck Surgery and Division of Molecular Biology and Human Genetics, the Medical Research Council, and Stellenbosch University. We gratefully acknowledge Dr Zubair Doorlakhan, who was involved with the initial study design, and the contributions of Ms Natalie Venema who recruited patients for the study, and Ms Megan Ferguson who conducted the audiological tests.

\section{References}

1. Wells CD, Cegielsk JP, Nelson LJ, et al. HIV infection and multidrug-resistant tuberculosis: the perfect storm. J Infect Dis 2007;196(Suppl 1): S86-S107. [http://dx.doi.org/10.1086/518665] [PMID:17624830] 2. World Health Organization. Guidelines for the Programmatic Management of Drug-resistant Tuberculosis. Emergency Update 2008. Geneva: World Health Organization, 2008. WHO/HTM TB/2008.402. http://whqlibdoc.who.int/publications/2008/9789241547581_eng.pdf (accessed 20 February 2010).

Selimoglu E. Aminoglycoside-induced ototoxicity. Curr Pharm 2007;13:119-126. [http://dx.doi org/10.2174/138161207779313731][PMID:17266591]

4. Moore RD, Smith CR, Lietman PS. Risk factors for the development of auditory ototoxicty in patients (eceiving a [PMID:6693788]

Bardien S, Human $\mathrm{H}$, Harris $\mathrm{T}$, et al. A rapid method for the detection of five known mutations associated with aminoglycoside induced deafness. BMC Med Genet 2009;10:2. http://www. biomedcentral.com/1471-2350/10/2 (accessed 30 March 2010).

6. Human H, Hagen CM, Bardien S, et al. Investigation of mitochondrial sequence variants associated with aminogloycoside-induced ototoxicity in South African TB patients on aminoglycosides. Biochem Biophys Res Commun 2010;393;751-756. [http:dx.doi.org/10.1086/j.bbrc.2010.02.075 [PMID:20171168] 
7. Tang HY, Hutcheson T, Neill S, et al. Genetic susceptibility to aminolgycoside ototoxicity: how many are at risk? Genet Med 2002;4(5):336-339. [http://dx.doi.org/10.1097/00125817-200209000-00004] [PMID:12394346].

8. Lu J, Qian Y, Li Z, et al. Mitochondrial haplotypes may modulate the phenotypic manifestation of the deafness associated 12S rRNA 1555A>G mutation. Mitochondrion 2010;10(1):69-81. [http:dx.doi. org/10.1016/j.mito.2009.09.007][PMID:19818876]

9. Duggal P, Sarkar M. Audiological monitoring of multi-drug resistant tuberculosis patients on aminoglycoside treatment with long term follow-up. BMC Ear Nose Throat Disorders 2007;7:2. http:// www.biomedcentral.com/1472-6815/7/5 (accessed 30 March 2010). [http://dx.doi.org/10.1186/1472 6815-7-5][PMID:17997841]

10. Torun T, Gungor G, Ozmen I, et al. Side effects associated with the treatment of multi-drug resistant tuberculosis. Int J Tuberc Lung Dis 2005;9(12):1373-1377. [PMID:16468160]
11. Khoza-Shangase K. Highly active antiretroviral therapy: Does it sound toxic? J Pharm Bioall Sci 2011;3:142-153. [http://dx.doi.org/10.4103/0975-7406.76494] [PMID:21430965]

2. Campbell K. Audiologic monitoring for ototoxicity. In: Roland P, Rutka J, eds. Ototoxicity. Hamilton, Canada: B C Decker Publishers, 2004:153-160.

13. Swart S. Draft Report: Modernisation of Audiology Sevices, Western Cape 2010. Cape Town: Department of Health, Western Cape, 2010:41-46.

14. Khoza-Shangase K, Mupawose A, Mlangeni NP. Ototoxic effects of tuberculosis treatments: How aware are patients? AJPP 2009;3(8):391-399. http://www.academicjournals.org/ajpp (accessed 7 March 2012)

Accepted 4 April 2012 